\title{
The anterior sylvian point and the suprasylvian operculum
}

\author{
Guilherme Carvalhal Ribas, M.D., Eduardo Carvalhal Ribas, \\ AND Consuelo Junqueira Rodrigues, M.D.
}

\author{
Clinical Anatomy Discipline of the Department of Surgery, University of São Paulo Medical School, \\ Hospital Albert Einstein, São Paulo, Brazil
}

\begin{abstract}
Object. The sylvian fissure or lateral sulcus is the most identifiable feature of the superolateral brain surface and constitutes the main microneurosurgical corridor, given the high frequency of approachable intracranial lesions through this route. The anterior sylvian point (ASyP) divides this fissure in its main anterior and posterior rami and was evaluated in this study for its morphology, exact location, and sulcal and neural relationships to assess its suitability as an initial, visually identifiable landmark for further neuroimaging and intraoperative estimation of its adjoining suprasylvian structures.

Methods. This study is based on 32 formalin-fixed cerebral hemispheres. The brains were removed from the skulls of 16 cadavers after the introduction of plastic catheters through properly positioned burr holes; the number of specimens for some of the analyzed data differed because of incorrect positioning of catheters or damage to the studied structures caused by the initial steps of the study.

The ASyP had a cisternal aspect in $94 \%$ of the specimens and was always located inferior to the triangular part of the inferior frontal gyrus, $2.3 \pm 0.5 \mathrm{~cm}$ in front of the inferior rolandic point. The ASyP was located underneath the 1.5 -cm-diameter cranial area of the anterior aspect of the squamous suture. Its adjoining structures that compose the suprasylvian operculum have constant basic morphological configurations.

Conclusions. The ASyP underlies the anterior aspect of squamous suture just behind the pterion, can be easily recognized, and constitutes a reliable initial sulcal landmark for further estimation of the suprasylvian sulcal and gyral structures. The suprasylvian operculum can be understood as a series of convolutions roughly arranged as a V-shaped convolution, with its vertex constituted by the ASyP, followed by three U-shaped convolutions and one C-shaped convolution.
\end{abstract}

\section{KeY WoRds - brain mapping - burr hole - cerebral cortex - prefrontal cortex - cranial suture • craniometry - craniotomy}

In their original description of the microsurgical anatomy of the subarachnoid cisterns in 1976, Yaşargil, et al., ${ }^{32}$ emphasized the importance of the SyF, which then became the main microneurosurgical corridor to the base of the brain. In later publications Yaşargil, et al., described in detail the microanatomy of this fissure and its underlying cistern ${ }^{29,30,33}$ and the technique of its opening. In agreement with other authors, ${ }^{12,14,16-18}$ Yaşargil divides the SyF into a proximal segment (stem, sphenoidal, anterior ramus) and a distal segment (lateral, posterior ramus) separated by the sylvian point, ${ }^{27,33}$ which is located beneath the triangular part of the IFG. The horizontal and the anterior ascending branches of the SyF that delineate the triangular part of the IFG arise at the sylvian point. ${ }^{14}$

Taylor and Haugton, ${ }^{23}$ in their study of the topography of the convolutions and fissures of the brain published in 1900 , used the term sylvian point, defining it as "the point where the main stem of the fissure of Sylvius reaches the

Abbreviations used in this paper: $\mathrm{ASqP}=$ anterior squamous point; $\mathrm{ASyF}=$ anterior sylvian fissure; ASyP = anterior sylvian point; IFG = inferior frontal gyrus; IFS = inferior frontal sulcus; $\mathrm{IRP}=$ inferior rolandic point; $\mathrm{SyF}=$ sylvian fissure. outer aspect of the hemisphere." In his textbook published in 1912, Krause $^{10}$ reproduced illustrations of the German anatomist August von Froriep (1849-1917) ${ }^{11}$ with identification of the sylvian point and also illustrated an anatomical opening of the SyF for the exposure of a superficial insular lesion (Fig. 1). Recently Türe, et al. ${ }^{27}$ stressed the use of the term sylvian point.

Despite the emphasis given by recent authors to the location of the sylvian point, none of them referred to the frequent enlargement of the fissure at the sylvian point, despite its presence in their own illustrations. ${ }^{12,14,16,27,29,30,33}$ This cisternal aspect of the sylvian point is also evident in the illustrations in many other recent publications ${ }^{4,8,13,15,18-22}$ and in old texts $10,24,25$ (Fig. 2).

The constant location and striking cisternal appearance of the sylvian point indicate that it can be used not only as a starting site to open the SyF, but also intraoperatively as an initial landmark to identify other important neural and sulcal structures along the fissure that are usually hidden by arachnoidal and vascular coverings. In this study the sylvian point is designated as the anterior as opposed to the posterior one,${ }^{26}$ which corresponds to the distal extremity of the posterior ramus of the SyF, from which the ascending 

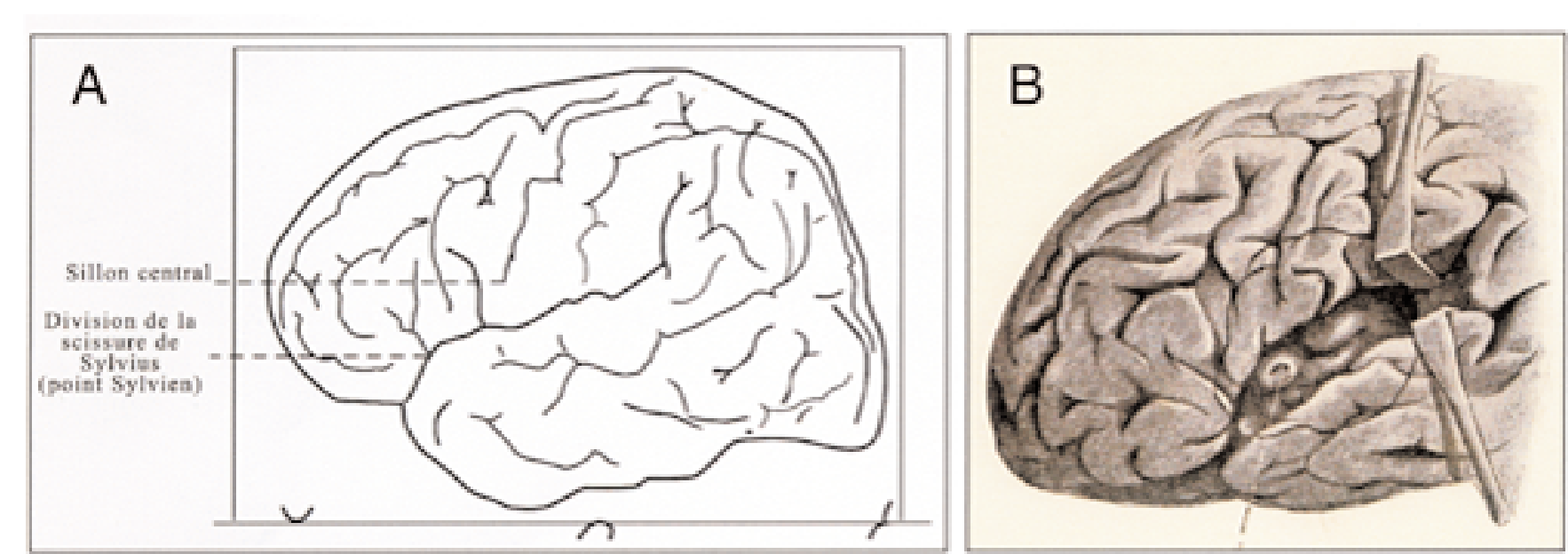

Fig. 1. Reproduction of an old illustration of the sylvian point by August von Froriep (A), and of the SyF opening by F. Krause (B). (Reprinted from Krause F: Chirurgie du Cerveau et de la Moelle Epiniere. Paris: Societé D’Editions Scientifiques et Medicales, 1912, Vols I and II.)

terminal ramus and the occasional descending terminal ramus originate..$^{14}$

Our aims in this study were as follows: 1) to evaluate and confirm the aforementioned general features of the anterior sylvian point; 2) to evaluate and establish the main relationships of the ASyP with other important neural and sulcal structures along the sylvian fissure; and 3) to establish the relationships of the ASyP with external cranial landmarks to orient its surgical exposure.

\section{MATERIALS AND METHODS}

Data were collected from 32 cerebral hemispheres obtained in 16 adult cadavers at the Death Verification Institute of the Department of Pathology and at the Clinical Anatomy Discipline of the Department of Surgery at the University of São Paulo Medical School, after authorization from the Ethical Committee for Analysis of Research Projects.
This study was conducted in two parts: Part I was a study of the surface anatomy of the ASyP and of its related neural and sulcal structures; and Part II was a study of the relationships of the ASyP with its external cranial landmarks. After proper identification of the cadaver and with the pathologist's consent, the study was conducted according to the following steps: 1) exposure of the external cranial surface by the standard biauricular necroscopic incision and detachment of both temporal muscles, with special attention paid to exposure of the cranial sutures in particular; 2) placement of a $1.5-\mathrm{cm}$ burr hole at the anterior part of the squamous suture, just behind the sphenoparietal suture, with an electrical drill (Dremel Moto-Tool; Dremel, Racine, WI); 3) opening of the dura mater with a No. 11 blade scalpel; 4) perpendicular introduction of an approximately 7-cm-long (2.5-mm-diameter) plastic tracheal aspiration tube (model sonda-suga No. 08; Embramed, São Paulo, Brazil) with the aid of a metallic guide; 5) circumferential opening of the skull with the appropriate saw and of the dura mater with
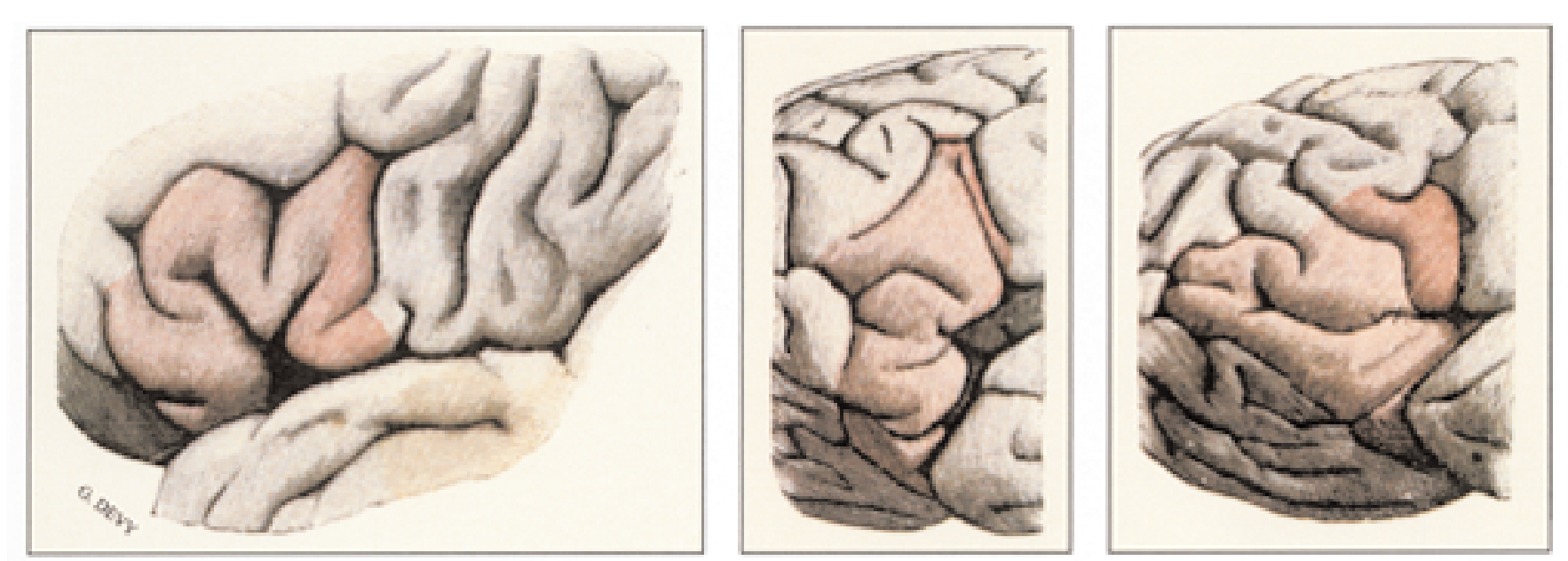

Fig. 2. Variations of the IFG illustrated by Testut and Jacob, showing the typical enlargement of the SyF beneath its triangular part. (Reprinted from Testut L, Jacob O: Tratado de Anatomia Topográfica, ed 5. Barcelona: Salvat, 1932.) 
scissors; 6) careful removal of the whole encephalon after basal divisions of the intracranial vessels and the cranial nerves; 7) replacement of the calvaria and closure of the scalp for necroscopic final procedures by the expert personnel; 8) storage of the resected encephalons in $10 \%$ formalin solution, with the specimens suspended by a string tied to the basilar artery to avoid brain deformations; 9) after adequate fixation (at least 2 months), section of the brainstem at the midbrain and its removal together with the cerebellum; 10) removal of the arachnoidal membranes and the superficial vessels of the cerebral hemispheres with the aid of microsurgical loupes (3.5×; Designs for Vision, Inc., Ronkonkoma, NY) and/or an operating microscope (model MDM; Carl Zeiss, Inc., Oberkochen, Germany); 11) microscopic evaluation and acquisition of measurements pertinent to the introduced catheter sites; and 12) further microscopic evaluation after removal of the catheters and acquisition of measurements relative to the neural and sulcal structures of interest for our study.

For didactic purposes, the first part of this study concerns the surface anatomy of the brain, whereas the second part deals with the relationship of the ASyP for cranial landmarks. Because some of the hemispheres were damaged by the catheter that was required for the second part of this study, the first part is based on fewer specimens than the second part. The measurements were recorded in millimeters and were completed with the aid of compasses and/or flexible millimetric tapes by the first author, at least twice.

\section{Statistical Analysis}

All continuous variables were summarized by the mean and standard deviation. For the evaluation of the cranialcerebral relationships, the 90th percentiles were also calculated.

\section{RESULTS: PART I}

\section{Surface Anatomy of the ASyP and the Suprasylvian Operculum}

Morphology and Location of the ASyP. The ASyP was located inferior to the triangular part and anterior/inferior to the opercular part of the IFG in all 18 specimens studied. Due to the usual retraction of the triangular part of the IFG in relation to the SyF, the ASyP was characterized as an enlargement of the SyF, with a mean diameter of 3 to $4 \mathrm{~mm}$ in nine specimens, larger than $5 \mathrm{~mm}$ in five specimens, between 2 and $3 \mathrm{~mm}$ in three specimens, and smaller than $2 \mathrm{~mm}$ in one specimen (Fig. 3).

Relationship of the ASyP With the IRP. The IRP was defined as the projection point of the inferior extremity of the central sulcus over the SyF, and the mean distance along the SyF between the ASyP and the IRP was $2.36 \pm 0.5 \mathrm{~cm}$.

\section{The Suprasylvian Operculum.}

The IFG Triangular Part. Despite its variable shape, the IFG triangular part was delimited anteriorly and posteriorly by the horizontal and anterior ascending rami of the $\mathrm{SyF}^{14}$ in all 20 specimens evaluated. The IFG triangular part was divided superiorly by a small sulcal segment of the IFS in 17 specimens, by a noncontinuous short IFS branch in two specimens, and one specimen contained no sulcal segment.

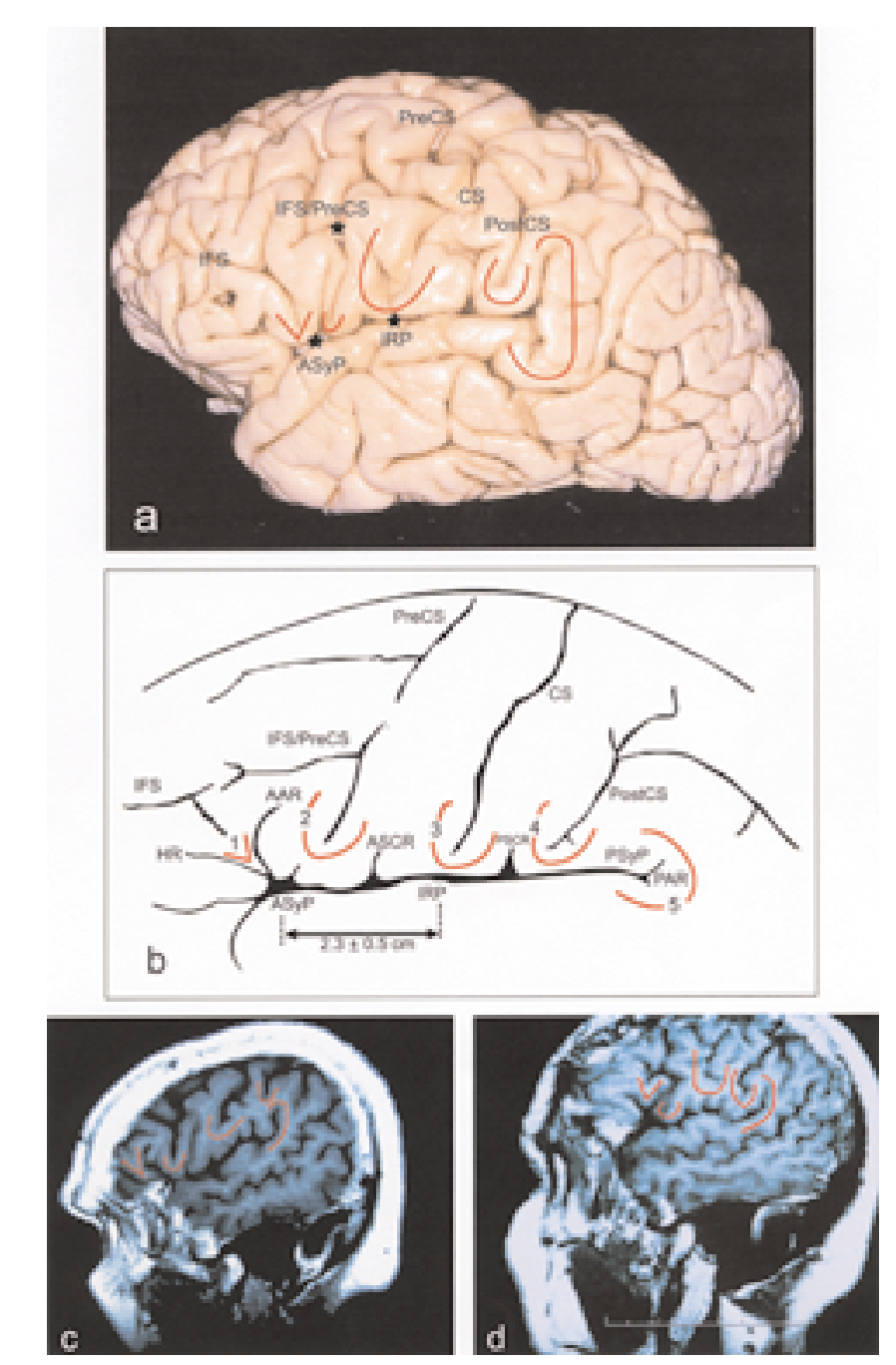

Fig. 3. A: Photograph of a cadaveric suprasylvian operculum specimen. B: Sketch of the neural and sulcal morphology (modified from Ono, et al.). C and D: Different magnetic resonance imaging sagittal views, with illustrations of the $\mathrm{V}$-shaped convolution (1) constituted by the triangular part of the IFG located just superiorly to the ASyP, and usually containing a descending branch of the IFS; of its following three U-shaped convolutions respectively comprised by (2) the opercular part of the IFG, which is always intersected by the inferior part of the precentral sulcus; the subcentral gyrus or rolandic operculum (3) composed by the inferior connection of the pre- and postcentral gyri enclosing the inferior part of the central sulcus; the connection arm (4) between the postcentral and supramarginal gyri that contains the inferior part of the postcentral sulcus; and finally the $\mathrm{C}$-shaped convolution (5) constituted by the connection of the supramarginal and superior temporal gyri that encircles the posterior end of the SyF. The bottoms of the U-shaped convolutions and their related sulcal extremities can be situated either superior to the fissure or inside its cleft. Stars designate the areas as labeled. AAR $=$ anterior ascending ramus of SyF; ASCR = anterior subcentral ramus; $C S=$ central sulcus; HR = horizontal ramus of SyF; IFS/PreCS = IFS and precentral sulcus meeting point; $\mathrm{PAR}=$ posterior ascending ramus of SyF; PostCS = postcentral sulcus; PreCS = precentral sulcus; $\mathrm{PSCR}=$ posterior subcentral ramus; $\mathrm{PSyP}=$ posterior sylvian point. 
The IFG Opercular Part. The IFG opercular part always had a $U$-shaped appearance. It was divided by the inferior segment of the precentral sulcus, and it was delimited anteriorly by the anterior ascending ramus and posteriorly by the anterior subcentral ramus of the SyF. The bottom of this $\mathrm{U}$-shaped convolution was superior and adjacent to the SyF in eight of the 18 specimens studied, and was enclosed inside the SyF in the 10 specimens in which the inferior extremity of the precentral sulcus was located at the level of or inside this fissure.

Rolandic Operculum. The posteriorly following inferior connection of the pre- and postcentral gyri (subcentral gyrus, rolandic operculum) also always presented as a $\mathrm{U}$ shaped convolution, which was contained inside the inferior segment of the central sulcus and was always delimited by the anterior and posterior subcentral sulci and the rami of the SyF. According to the position of the inferior extremity of the central sulcus in relation to the SyF, the bottom of the $U$ was superior and adjacent to the SyF in 25 of the 30 specimens studied and enclosed inside the SyF in the other five.

Postcentral and Supramarginal Gyri Connection. The postcentral and supramarginal gyri connection arm was always present and was disposed between the posterior subcentral ramus and the posterior ascending ramus of the $\mathrm{SyF}$ and was also configured as a $U$-shaped convolution contained inside the inferior segment of the postcentral sulcus in the 18 specimens. The bottom of the $U$ was found to be superior to the SyF in 11 of the 18 specimens and inside the SyF in the other seven, according to the positioning of the inferior extremity of the postcentral sulcus in relation to the SyF. In all 18 specimens, the supramarginal gyrus was continuous with the superior temporal gyrus through a C-shaped gyral connection that encircled the distal end of the SyF.

\section{RESULTS: PART II}

\section{Relationship of the ASyP With the External Cranial Surface}

The relationship of the ASyP with the external cranial surface was evaluated based on the study of topographic correlations between the ASyP and a skull point that was designated as the ASqP. The ASqP was defined as the central point of a $1.5-\mathrm{cm}$-diameter burr hole located on the most anterior segment of the squamous suture, superior to the sphenosquamous suture and just posterior to the sphenoparietal suture, and hence over the squamous suture just posterior to the $\mathrm{H}$-shaped central bar that characterizes the pterion.

Pterion and ASqP Identification. After its exposure, the pterion had an evident $\mathrm{H}$-shaped morphology in 23 of the 32 specimens, and it had a dissimilar shape in the other nine, but allowed an easy and proper ASqP identification in all specimens studied. $A S y P$

Topographic Relationships Between the ASqP and the

Vertical Relationship. The ASqP was superior to the ASyP in one of the 27 specimens studied, was situated at the ASyP level in 19, and was inferior to the ASyP in the other seven, at a mean distance of $0.18 \pm 0.41 \mathrm{~cm}$ inferior to the ASyP. The 90th percentile for probability values pertinent to the vertical positioning of the $\mathrm{ASqP}$ in relation to the ASyP (total $0.00 \mathrm{~cm}$, superior $0.00 \mathrm{~cm}$, inferior 0.00 $\mathrm{cm}$ ) disclose the very close vertical relationship between the ASyP and the squamous suture.

Horizontal Relationship. The ASqP was anterior to the ASyP in six of the 27 specimens, at the same level as the ASyP along the SyF in 15, and posterior to the ASyP in the other six specimens, with a mean distance of $0.02 \pm 0.53$ $\mathrm{cm}$ anterior to the ASyP. The 90th percentile for probability values pertinent to the horizontal positioning of the $\mathrm{ASqP}$ in relation to the ASyP (total $0.68 \mathrm{~cm}$, anterior 0.00 $\mathrm{cm}$, posterior $0.92 \mathrm{~cm}$ ), disclosed a slight predominance of anterior positioning of the ASyP in relation to the ASqP.

\section{DISCUSSION}

The sylvian fissure is the single most identifiable feature of the superolateral face of the brain, and together with the underlying sylvian cistern it constitutes the most frequently used microneurosurgical corridor because of the high proportion of intracranial lesions that are accessible through its opening. The transsylvian approach ${ }^{32}$ through a pterional craniotomy $y^{31,33}$ is particularly useful for anterior basal extrinsic lesions and for frontobasal, mesial temporal, and insular intrinsic intracranial lesions..$^{29,30,33}$ Other frontotemporal craniotomies derived from the pterional ${ }^{29,31}$ and

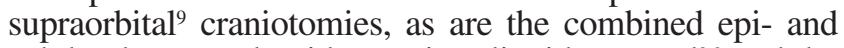
subdural approach with anterior clinoid removal ${ }^{2,3}$ and the orbitozygomatic extension of the pterional craniotomy, ${ }^{6,7}$ enhance the basal approaches and minimize the brain retraction but do not disregard the opening of the SyF to optimize its ideal exposures.

Our findings relative to the ASyP indicate that it constitutes an easily identifiable sulcal landmark with consistent topographic relationships. Hence, it constitutes a good microneurosurgical starting point for anatomical orientation and for the SyF opening.

The superior and inferior margins of the SyF constitute the frontoparietal and temporal operculi, which cover the superior and inferior aspects of the insula. ${ }^{27}$ The frontoparietal operculum extends from the anterior to the posterior ascending branch of the $\mathrm{SyF}^{28}$ With the orbital part of the IFG disposed anteriorly, the suprasylvian structures can be understood as a series of convolutions roughly arranged in a V shape with its vertex constituted by the ASyP, followed by three $\mathrm{U}$-shaped convolutions and one $\mathrm{C}$-shaped convolution. The bottoms of the three U-shaped convolutions and their related sulcal extremities can be situated either superior to the SyF or inside it, thereby giving the false visual impression that their related sulci end at the SyF.

The anterior V-shaped convolution is constituted by the triangular part of the IFG and is located just superior to the ASyP. The horizontal and the anterior ascending rami of the SyF delineate this convolution. Usually the triangular part of the IFG contains a descending branch of the inferior frontal sulcus (IFS).

The most anterior $U$-shaped convolution is the opercular part of the IFG, which encloses the inferior aspect of the precentral sulcus. In agreement with Ebeling, et al., 5 and Ono, et al., ${ }^{14}$ we also found that the precentral sulcus always ends inside the opercular part of the IFG, superiorly 
or adjacent to the SyF (44\%) or inside the SyF (56\%). The $U$-shaped convolution that encloses the inferior segment of the precentral sulcus corresponds anteriorly to the opercular part itself and posteriorly to its connection with the precentral gyrus. Anteriorly the opercular part of the IFG is delimited by the anterior ascending ramus of the SyF, and posteriorly by the anterior subcentral ramus of the SyF. Together, the triangular and opercular parts of the IFG constitute the motor speech area of Broca in the dominant hemisphere ${ }^{1,8}$ and the intraoperative estimation of its localization can then be done based on the initial identification of the ASyP.

The middle U-shaped convolution is called the subcentral gyrus, and it connects the precentral and postcentral gyri. The subcentral gyrus is also called the inferior frontoparietal plis de passage of Broca ${ }^{1}$ or the rolandic operculum, because it encircles the inferior part of the central sulcus. The location of the subcentral gyrus in relation to the SyF varied in accordance with the positioning of the IRP; it could be found either superior or adjacent to the SyF (83\%) or inside it (17\%). The subcentral gyrus is delimited by the anterior and posterior subcentral rami of the SyF.

The third U-shaped convolution, which is composed of the arm connecting the postcentral and supramarginal gyri, contains the inferior part of the postcentral sulcus. It is delimited anteriorly by the posterior subcentral ramus of the SyF, and posteriorly by the posterior ascending ramus of the SyF. According to the position of the inferior extremity of the postcentral sulcus in relation to the SyF, the bottom of this third U-shaped convolution can be superior to the SyF $(61 \%)$ or inside it $(39 \%)$.

The C-shaped convolution that completes the frontoparietal or suprasylvian operculum is constituted by the connection arm between the supramarginal gyrus and the superior temporal gyri, which encircles the posterior end of the SyF. The inferior margin of the SyF is related only to the superior temporal gyrus that constitutes the temporal operculum.

Aside from its usually evident cisternal aspect due to the fact that the IFG triangular part is generally retracted in relation to its adjacent anterior orbital and posterior opercular parts, the ASyP can also be intraoperatively identified as the SyF segment located just posterior to the IFG orbital part once this convolution bulges after the dural opening.

Yaşargil also emphasizes that "the Sylvian point is located in the same plane of the IFG triangular part, and 10 to $15 \mathrm{~mm}$ anterior to the Sylvian venous confluence constituted by frontal and temporal tributaries veins," and advises "to begin opening the fissure immediately anterior to this vein confluence at a point where a temporal or frontal artery or where both arteries appear at the surface of the fissure, ${ }^{33}$ hence at the ASyP area. The opening of this fissure site soon exposes the insular apex, ${ }^{26}$ and the limen insula and the middle cerebral artery bifurcation are located a little deeper and more anterior, 10 to $20 \mathrm{~mm}$ perpendicular to the ASyP itself. ${ }^{33}$

Whereas the opening of the SyF posterior to the ASyP exposes the lateral aspect of the insula, the opening of its stem anterior to the ASyP leads to the suprasellar cisterns. In conjunction with the opening of the sylvian ascending ramus, this exposes the anterior periinsular sulcus, ${ }^{26}$ and thus the anterior aspect of the insula situated behind the posterior orbital gyrus.

In regard to the ASyP projection on the external cranial surface, Taylor and Haugton (1900) ${ }^{23}$ described it in the past as corresponding to the point of intersection of the sylvian line, constituted by a line from the junction of the third and fourth segments of the nasion-inion curvature to the orbitotemporal angle, with the line drawn from the junction of the first and second segments of the nasion-inion curvature to the external auditory meatus.

The consistently short distance between the ASqP and the ASyP indicates that the ASyP is related to the $1.5-\mathrm{cm}-$ diameter burr hole centered on the anterior segment of the squamous suture just behind the pterion (Fig. 4).

The knowledge of the ASyP distance to the IRP along the SyF allows the neurosurgeon to estimate visually the projection of the central sulcus over the SyF. The distance of $2.3 \pm 0.5 \mathrm{~cm}$ is in accordance with the findings of Ono, et al. ${ }^{14}$

The knowledge of these basic anatomical features and relationships can then lead us to a relatively precise initial visual identification of the ASyP, both in preoperative neuroimaging studies and intraoperatively, and from it to fur-
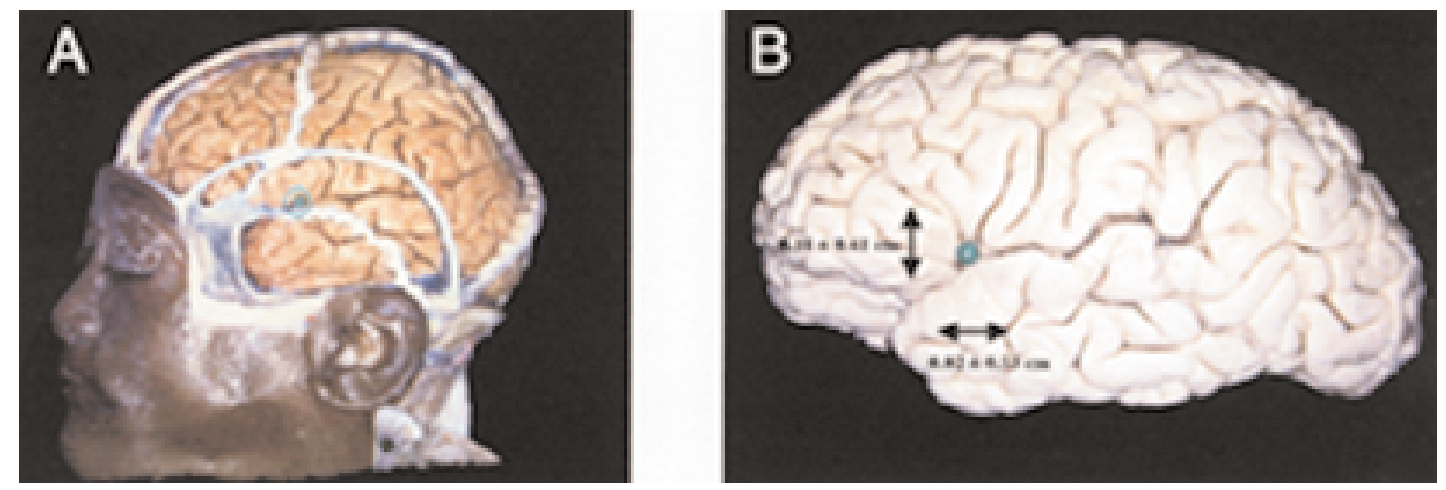

Fig. 4. A: Photograph obtained at necropsy showing a 1.5-cm-diameter area (green ball) over the anterior segment of the squamous suture, just behind the pterion. B: Photograph of cadaveric brain showing the horizontal and vertical mean distances of the ASyP from the central point of the anterior squamous suture in a 1.5 -cm-diameter area. 
ther sulcal and gyral recognition, simplifying the tridimensional understanding of the intracranially related structures and lesions and the proper placement of related craniotomies.

\section{CONCLUSIONS}

Given its consistent morphological features and cranial and neural relationships, the ASyP can be exposed and visually identified with a significant degree of assurance. It constitutes an adequate initial landmark for further estimation of the positioning of other opercular sulcal and gyral structures along the SyF.

\section{References}

1. Broca P: Sur la topographie crânie-cerébrale ou sur les rapports anatomiques du crâne et du cerveau. Rev Anthrop 5:193-248, 1876

2. Dolenc VV: A combined epi-and subdural direct approach to carotidophthalmic artery aneurysms. J Neurosurg 62:667-672, 1985

3. Dolenc VV, Yaşargil MG: Anatomy and Surgery of the Cavernous Sinus. Wien: Springer-Verlag, 1989

4. Duvernoy HM: The Human Brain. Wien: Springer, 1991

5. Ebeling U, Steinmetz H, Huang Y, et al: Topography and identification of the inferior precentral sulcus in MR imaging. AJNR 10:101-107, 1989

6. Fujitsu K, Kiwabara T: Zygomatic approach for lesions in the interpeduncular cistern. J Neurosurg 62:340-343, 1985

7. Hakuba A, Liu S, Nishimura S: The orbitozygomatic infratemporal approach: a new surgical technique. Surg Neurol 26: 271-276, 1986

8. Heimer L: The Human Brain an Spinal Cord: Functional Neuroanatomy and Dissection Guide, ed 2. New York: Springer Verlag, 1995

9. Jane JA, Park TS, Pobereskin LH, et al: The supraorbital approach: technical note. Neurosurgery 11:537-542, 1982

10. Krause F: Chirurgie du Cerveau et de la Moelle Epiniere. Paris: Societé D'Editions Scientifiques et Medicales, 1912

11. Lockard I: Desk Reference for Neuroanatomy: a Guide to Essential Terms. New York: Springer-Verlag, 1977

12. Naidich TP, Valavanis AG, Kubik S: Anatomic relationships along the low-middle convexity: Part I-normal specimens and magnetic resonance imaging. Neurosurgery 36:517-532, 1995

13. Nieuwenhuys R, Voogd J, Van Huijzen C: The Human Central Nervous System, ed 3. Berlin: Springer-Verlag, 1988

14. Ono M, Kubik S, Abernathey CD: Atlas of Cerebral Sulci. Stuttgart: Thieme, 1990

15. Pernkoff E: Atlas of Topographical and Applied Human Anatomy. Baltimore: Urban \& Schwarzenberg, 1989
G. C. Ribas, E. C. Ribas, and C. J. Rodrigues

16. Rhoton AL Jr: The supratentorial cranial space: microsurgical anatomy and surgical approaches. Neurosurgery 51 (Suppl 4): S1-S298, 2002

17. Seeger W: Atlas of Topographical Anatomy of the Brain and Surrounding Structures for Neurosurgeons, Neuroradiologists, and Neuropathologists. Wien: Springer, 1978

18. Seeger W: Microsurgery of the Brain. Anatomical and Technical Principles. Wien: Springer, 1980

19. Seeger W: Differential Approaches in Microsurgery of the Brain. Wien: Springer, 1985

20. Seeger W: Microsurgery of Intracranial Tumors. Wien: Springer-Verlag, 1995

21. Squire LR, Bloom FE, McConnell SK, et al: Fundamental Neuroscience, ed 2. Amsterdam: Academic Press, 2003

22. Tamraz JC, Comair YG: Atlas of Regional Anatomy of the Brain Using MRI. Berlin: Springer, 2000

23. Taylor EJ, Haugton WS: Some recent researches on the topography of the convolutions and fissures of the brain. Trans $\mathbf{R}$ Acad Med Ireland 18:511-519, 1900

24. Testut L, Jacob O: Tratado de Anatomia Topográfica, ed 5. Barcelona: Salvat, 1932

25. Testut L, Latarjet A: Tratado de Anatomia Humana, ed 8. Barcelona: Salvat, 1932

26. Tirakotai W, Sure U, Benes L, et al: Image-guided transsylvian, transinsular approach for insular cavernous angiomas. Neurosurgery 53:1299-1305, 2003

27. Türe U, Yaşargil DCH, Al-Mefty O, et al: Topographic anatomy of the insular region. J Neurosurg 90:720-733, 1999

28. Williams PL, Warwick R (eds): Gray's Anatomy, ed 36. Philadelphia: Saunders, 1980

29. Yaşargil MG: Microneurosurgery. Stuttgart: Georg Thieme, 1984, Vol I

30. Yaşargil MG: Microneurosurgery. Stuttgart: Georg Thieme, 1994, Vol IV

31. Yaşargil MG, Fox JL, Ray MW: The operative approach to aneurysms of the anterior communicating artery, in Krayenbül $\mathrm{H}$ (ed): Advances and Technical Standards in Neurosurgery. Wien: Springer-Verlag, 1975, Vol 2, pp 114-170

32. Yaşargil MG, Kasdaglis K, Jain KK, et al: Anatomical observations of the subarachnoid cisterns of the brain during surgery. J Neurosurg 44:298-302, 1976

33. Yaşargil MG, Krisht AF, Türe U, et al: Microsurgery of insular gliomas: Part I, II, IV. Contemporary Neurosurgery 24:(11):1-8; (13):1-6; (14):1-8, 2002

Manuscript received March 28, 2005.

Accepted in final form May 26, 2005.

Address reprint requests to: Guilherme Carvalhal Ribas, M.D., University of São Paulo Medical School, Hospital Albert Einstein, R Eduardo Monteiro 567,Sao Paulo, Brazil 05614-120. email: guilherme @ ribas.med.br. 